\title{
INNOVATIVE DESIGN OF THE SEAL STRUCTURE OF BUTTERFLY VALVE BASED ON TRIZ
}

\author{
Jianhui Zhang ${ }^{1}$, Runhua Tan ${ }^{1}$, Ping Jiang ${ }^{1}$, Jinling Dai ${ }^{2}$ \\ 'Institute of Design for Innovation, Hebei University of Technology, Tianjin, 300130, PRC; \\ Email: zjh2031@sina.com. ${ }^{2}$ Institute of Pharmaceutical Research, Tianjin, 300073, PRC.
}

\begin{abstract}
Innovative ideas generation is a key step in the innovative design of the products. Computer-aided innovation (CAI) systems based on TRIZ (Theory of inventive problem solving) support new ideas generation of innovation. The patterns and lines of technology evolution in TRIZ provide a powerful structured methodology for a directed development of new products. The concept of 'evolutionary potential' and the method to construct an evolutionary potential plot are introduced. The Innovative ideas for new product development can be generated by the analysis of the evolutionary potential. A general process of the innovative ideas generation is then developed. A butterfly valve's seal structure case study is presented to demonstrate the process.
\end{abstract}

Key words: TRIZ; patterns and lines of technology evolution; evolutionary potential; innovative idea generation; butterfly valve.

\section{INTRODUCTION}

Butterfly valve is a controlling valve used in large-size pipe, which has function of opening or closing pipe, regulating flux and check, etc. With the development of the industry, the demand for the valve capability becomes stricter and stricter. So the design of the seal structure of the butterfly valve has been an important research task at all times.

The theory of technology evolution in TRIZ (Theory of inventive problem solving) provides the powerful tools of technological forecastingpatterns of evolution and lines of evolution, which can forecast the

Please use the following format when citing this chapter:

Zhang, Jianhui, Tan, Runhua, Jiang, Ping, Dai, Jinling, 2006, in International Federation for Information Processing (IFIP), Volume 207, Knowledge Enterprise: Intelligent Strategies In Product Design, Manufacturing, and Management, eds. K. Wang, Kovacs G., Wozny M., Fang M., (Boston: Springer), pp. 450-456. 

TRIZ

probabilities of certain design parameters falling within particular confidence intervals at some future time (Alla, et al. 2002). Evolutionary potential can be found when patterns and lines of technological evolution, which match the historical evolution of the core technology of the products, are applied to analyze the products. And the innovative ideas for future technological R\&D plans can be generated by analysis of the evolutionary potential. The computer-aided innovation (CAI) systems based on TRIZ are practical tools to support this process.

This paper introduces a general process of the innovative ideas generation supported by computer-aided innovation (CAI) systems. A butterfly valve's seal structure case study is presented to demonstrate the process step by step.

\section{TECHNOLOGY EVOLUTION AND EVOLUTIONARY POTENTIAL}

Patterns of technical system evolution are revealed by decades of analysis of hundreds of thousand of the global inventive patent. The patterns define general technological evolutional directions and each pattern includes multiple lines of evolution that specify particular evolutional stages or scenarios along these directions (Altshuller, 1984; Tan, 2002).

By comparing the products under study with each of the lines and making a connection with one line stage, the state of the lines that can best describe the current evolutionary state of the design defined as 'current state', and the unused states are defined as 'potential states'. The 'evolutionary potential' concept is a means of observing the relevant evolution lines of product together (Darrell, 2002). Fig. I illustrates the structure of evolutionary potential plot. An evolution line known as 'space segmentation' in the figure describes the evolution of the use of space inside structures. There are five states shown in this line, the distance between the ending state and the current state in the line exists the 'evolutionary potential'.

If a system is under evaluation relative to the 'space segmentation' line, a state of the art design will be at stage 3 of 5 possible stages. Repeating this process for each line in turn, then arranging each line as one spoke on a radar plot reveal a picture of the overall current evolutionary state of the product. As shown in Fig. 1, the shaded area of the radar plot represents how far along each line the current system has evolved, the area difference between shaded area and perimeter is a measure of untapped 'evolutionary potential'. The evolution lines and the untapped potential act as signposts indicating the directions that other successful systems have evolved in. So the new ideas 
generated by analysis of the potential states are more reliable than that generated hy traditinnal methode

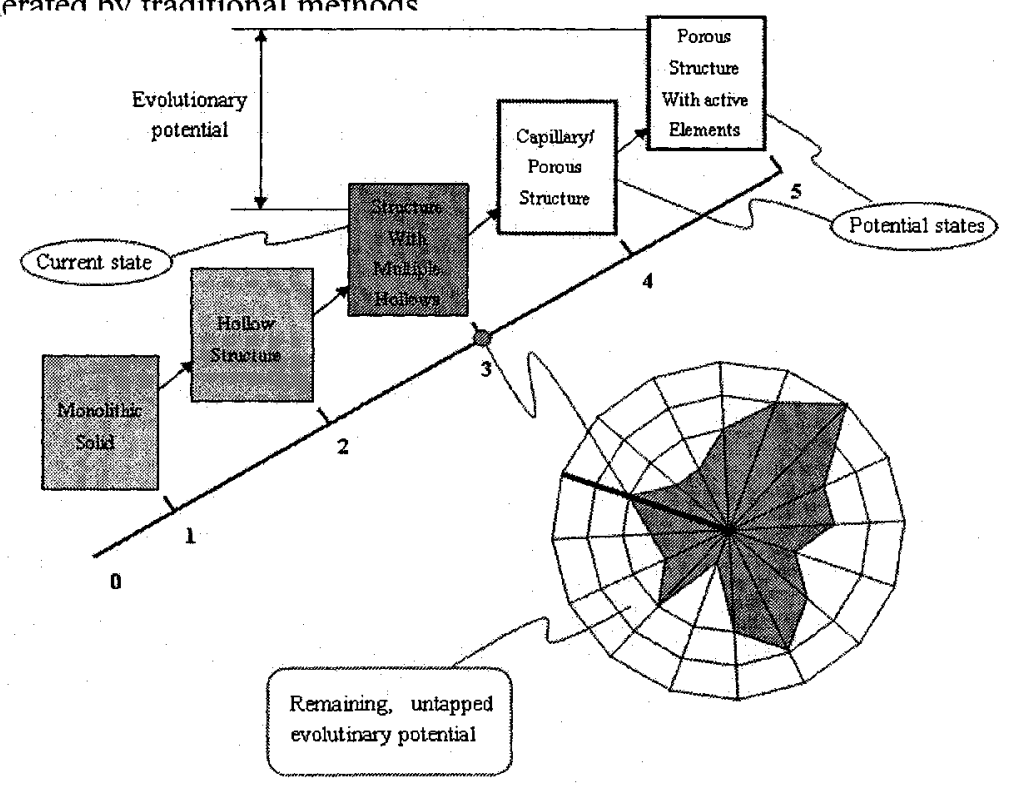

Figure 1. Evolutionary potential radar plot structure

\section{A GENERAL PROCESS OF INNOVATIVE IDEAS GENERATION USING CAI SYSTEM}

The technological system evolution is propelled by core technical innovation, and the key patents about a certain product are the track record of core technical innovation of the product and can represent the history of product evolution. So the patent information of the product is treated as the main source of product evolution analysis. The general process of the innovative ideas generation includes the following six steps:

Step 1: Analyze the history of product evolution

By searching and analyzing the relevant key patent of the products, valuable technical and business information regarding target or key technology of the product can be available.

Step 2: Apply the relevant patterns and lines to analyze the product

By comparing the product under study with each of the selected patterns and lines of CAI system, and making a connection with one evolution state, the current state and the potential states for the product are determined. The 
TRIZ

CAI systems, such as Goldfire Innovator of Invention Machine (USA) and InventionTool (China) support this step.

Step 3: Draw the evolutionary potential radar plot

On the basis of the analysis of step 2, trace out the product's evolutionary potential radar plot.

Step 4: Search evolutionary potential

Evolutionary potential can be found from one or more lines selected in the evolutionary potential radar plot. InventionTool (China) supports step 3 and step 4.

Step 5: Generate innovative ideas

Under analyzing the potential line in the evolutionary potential radar plot, one or more innovative ideas can be generated.

\section{INNOVATIVE IDEA GENERATION AND APPLICATION FOR BUTTERFLY VALVE'S SEAL STRUCTURE}

The innovative idea generation and application for the butterfly valve is carried out using the general process step by step.

Step 1: Analyze the history of product evolution

Search the butterfly valve patents of different industry in Chinese patent databases and study tends of the technology evolution for the main structure of the valves. A butterfly valve is divided into three modules: the controlling module (includes drive and transmission section), executing module (as shown in Fig. 2, (4), includes valve disc, valve shaft, valve seat, and seal ring) and valve body. The evolution of the executing module shows the core technology changes of this kind of valve. The evolutional tree of the executing module of butterfly valve is shown in Fig. 2 (Zhang, 2004).

In order to clearly show the evolution of various generations of butterfly valve systems, we define the generations of butterfly valve as follows: the first generations -(1), the second generations - (2), (3), (4), the third generations -(5),(6), (7), (8) and the fourth generations -(9), (10). The same generation products share some common features. For example, the offset between shaft and disc has transformed from zero to single, double and triple, as shown in fig. $2($ (1) $\rightarrow$ (3) $\rightarrow$ (7) $\rightarrow$ (10).

Step 2: Apply the relevant patterns and lines to analyze the product

Applying the patterns and lines that match the evolution of butterfly valve to analyze the main structure of butterfly valve, there are eleven lines has been used in the evolution of the butterfly valve. The current state and 
the potential states for the product are determined. As shown in Fig. 3. These lines are selected from Goldfire Innovator 4.0 of Invention Machine (USA)

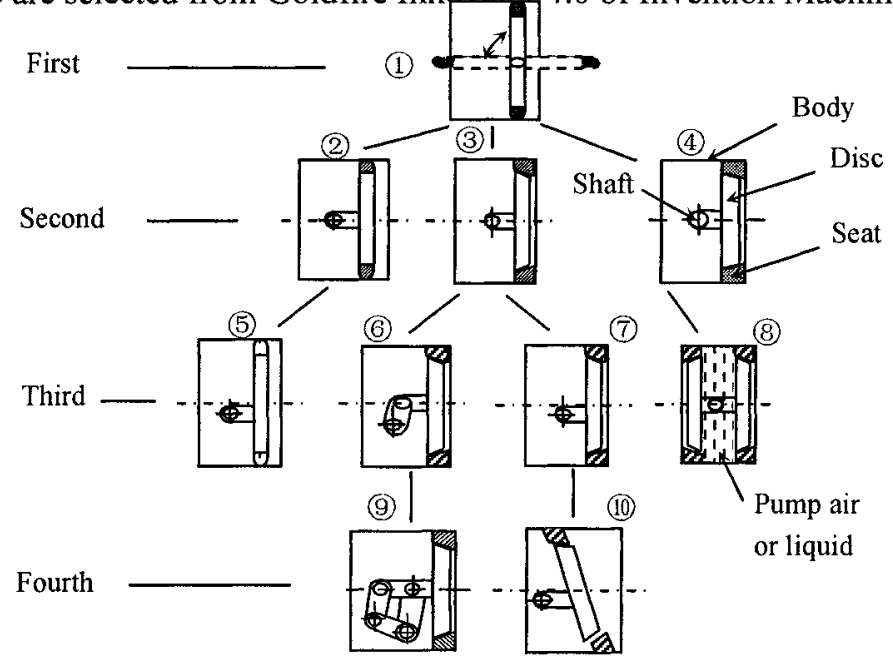

Figure 2. The evolution tree of executing module structure of butterfly valve

and InventionTool 3.0 (China).

Step 3: Draw the evolutionary potential radar plot

In order to see how quickly the evolutionary potential is being used by the various generations of design of butterfly valve systems, overlaying the evolutionary potential plots for each generation, a composite picture highlighting the evolution process that has taken place from the first generation butterfly valve to the most recent is illustrated in Fig. 3. The plot labeled ' 1 ', ' 2 ', ' 3 ' and ' 4 ' describes the evolutionary state of the four generations respectively. InventionTool 3.0 (China) supports this step and the next step.

Step 4: Search evolutionary potential

As shown in Fig. 2, undoubtedly butterfly valve technology may be seen to be mature in terms of dimension and geometric structure evolutionbecause all of the available dimensional freedoms have been used. Beyond that, however, the plot clearly shows there is still considerable potential in the area difference between shaded area and perimeter. Therefore, there are consequently significant improvements that can be developed.

Many butterfly valve patents have related to sealed issues, in order to improve the integral leakage index. On the basis of the above analysis, evolution line of 'increased dynamics' was selected to find the evolutionary 
TRIZ

potential in relation to the seal technology of the butterfly valve. There are six states in this line:

\section{Dynamics}

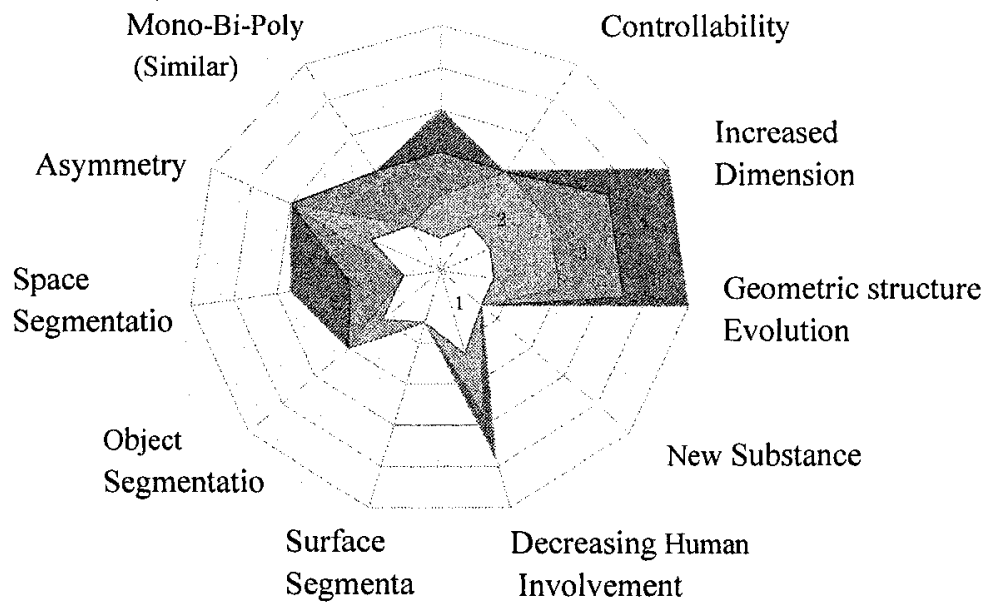

Figure 3. Evolutionary history of butterfly valve technology

stiff system $\rightarrow$ a joint $\rightarrow$ many joints $\rightarrow$ elastic $\rightarrow$ molecular (liquid, gas) $\rightarrow$ field

The current state is "elastic", such as the application of elastic material of seal ring. The states of "molecular" and "field" are potential states.

Step 5: Generate innovative ideas

The key technique of the valve is to compensate for the wear of the seal ring in order to reduce leakage. The action of the compensation can be achieved by making the valve disc to move forward in a small displacement after the rotation of the valve disc when closing the valve, or making the valve disc to move backward in a small displacement before the rotation of the valve disc when opening the valve.

The application of the elastic elements in current state can implement the function above-mentioned. For example, the elasticity of the spring can implement that function from the current state "elastic", the compressibility of liquid or gas can implement that function from the first potential state "molecular", the second potential state is also applicable. For example, a permanent magnet can implement the function of the compensation. So, some innovative ideas are generated.

Innovative ideas: Apply elasticity of spring, or compressibility of liquid or gas, or a permanent magnet to implement the function of the seal ring compensation. 
These three innovative ideas should be possible because there are successful applications in industry or life. These new ideas are generated from the 'evolutionary potential' that other successful industries have already made, so the uncertainty of the new ideas is reduced. The engineers of the butterfly valve industry should also make evaluation for the ideas and implement them by the conditions of management, design and manufacture capacity of their firm, they should make an adaptive and specific design for the existing products that need to be improved. Accordingly, the pace of the products innovation is accelerated.

\section{SUMMARY}

To generate innovative ideas is a key step in the innovative design of the products. CAI systems based on TRIZ are applicable to support this process. A general process of the innovative ideas generation supported by computeraided innovation (CAI) systems is developed.

Innovative design of seal structure of butterfly valve is carried out and shows the general process of the innovative generation and application step by step. Although perhaps thought of as 'mature' technologies, the valve still have considerable levels of untapped evolutionary potential remaining, and that there are consequently significant opportunities for future development of it.

\section{ACKNOWLEDGMENT}

The research is supported in part by Natural Science Foundation of China under Grant Numbers 50375045 and TianJin Natural Science Foundation Key Projects under Grant Number 043802211.

\section{REFERENCES}

1. Altshuller, G.S., 1984, Creativity As An Exact Science: The Theory of the Solution of Inventive Problems, The United Kingdom: Gordon and Breach Science Publishers Inc., London, pp. 205-216.

2. Tan Runhua, 2002, Innovation Design-TRIZ: Theory of Inventive Problem Solving, China Machine Press, Beijing, pp. 58-60 (in Chinese).

3. Alla, Z., Boris, Z., and Gafur, Z., (June 2001); An Application of Directed Evolution, http://www.Ideation-triz.com.

4. Darrell Mann, 2002, Evolutionary-PotentialTM in Technical and Business Systems, TRIZ Journal, 6, http://www.triz-journal.com.

5. Zhang, H.G., Tan, R.H., and Zhao, W.Y., 2004, The technology maturity of product and its mapping, ASME Proceedings of IMECE'04, IMECE2004-59260. Anaheim, USA. 